\title{
Targeting of micelles and liposomes loaded with the pro- apoptotic drug, NCL-240, into NCI/ADR-RES cells in a 3D spheroid model
}

\author{
Bhushan S. Pattni ${ }^{1}$, Srikar G. Nagelli ${ }^{1}$, Bhawani Aryasomayajula ${ }^{1}$, Pranali P. Deshpande ${ }^{1}$, \\ Abhijit Kulkarni ${ }^{1}$, William C. Hartner ${ }^{1}$, Ganesh Thakur ${ }^{1}$, Alexei Degterev ${ }^{2}$, and Vladimir P. \\ Torchilin 1,3 \\ ${ }^{1}$ Department of Pharmaceutical Sciences, Center for Pharmaceutical Biotechnology and \\ Nanomedicine, Northeastern University, Boston, MA, USA \\ ${ }^{2}$ Department of Biochemistry, Tufts University School of Medicine, Boston, MA, USA \\ ${ }^{3}$ Department of Biochemistry, Faculty of Science, King Abdulaziz University, Jeddah 21589, Saudi \\ Arabia
}

\begin{abstract}
Purpose-To develop transferrin (Tf)-targeted delivery systems for the pro-apoptotic drug, NCL-240, and to evaluate the efficacy of this delivery system in ovarian cancer NCI/ADR-RES cells, grown in vitro in a 3D spheroid model.
\end{abstract}

Methods-Tf-targeted PEG-PE-based micellar and ePC/CHOL-based liposomal delivery systems for NCL-240 were prepared. NCI/ADR-RES cells were used to generate spheroids by a non-adhesive liquid overlay technique. Spheroid growth and development were monitored by size (diameter) analysis and H\&E staining. The targeted formulations were compared to untargeted ones in terms of their degree of spheroid association and penetration. A cell viability analysis with NCL-240-loaded micelles and liposomes was performed to assess the effectiveness of Tftargeting.

Results-Tf-targeted polymeric micelles and Tf-targeted liposomes loaded with NCL-240 were prepared. NCI/ADR-RES cells generated spheroids that demonstrated the presence of a distinct necrotic core along with proliferating cells in the spheroid periphery, partly mimicking in vivo tumors. The Tf-targeted micelles and liposomes had a deeper spheroid penetration as compared to the untargeted delivery systems. Cell viability studies using the spheroid model demonstrated that Tf-mediated targeting markedly improved the cytotoxicity profile of NCL-240.

Conclusion-Transferrin targeting enhanced delivery and effectiveness of micelles and liposomes loaded with NCL-240 against NCI/ADR-RES cancer cells in a 3D spheroid model.

\section{Keywords}

NCL-240; cancer spheroids; micelles; liposomes; transferrin-targeted drug delivery

Corresponding author: Vladimir Torchilin: v.torchilin@ neu.edu.

Current Address: 140 The Fenway, Room 236, Department of Pharmaceutical Sciences, Center for Pharmaceutical Biotechnology and Nanomedicine, Northeastern University, 360 Huntington Avenue, Boston, MA 02115, United States of America. 


\section{INTRODUCTION}

A common feature observable in multiple cancer cell lines is the activation of the phosphatidylinositol 3-kinase (PI3K) pathway [1, 2]. The activation of the PI3K pathway via enhanced release of growth factors initiates a signaling cascade beginning with the phosphorylation of phosphatidylinositol-3,4-diphosphate ( $\left.\mathrm{PIP}_{2}\right)$ to a second messenger phosphatidylinositol-3,4,5-triphosphate $\left(\mathrm{PIP}_{3}\right)$. $\mathrm{PIP}_{3}$ is responsible for activating several proteins through pleckstrin homology $(\mathrm{PH})$ binding and consequently mediates multiple cellular functions via the Akt/mTOR pathway responsible for survival, growth and motility. The protein phosphatase and tensin homolog (PTEN) is a negative regulator via dephosphorylation of $\mathrm{PIP}_{3}$ to $\mathrm{PIP}_{2}$ that interrupts the PI3K pathway. A gain in activity of $\mathrm{Akt} / \mathrm{PIP}_{2}$ or a loss of function of PTEN manifest in cancerous cells results in an amplified $\mathrm{PIP}_{3}$ and subsequent promotion of cancer cell survival. This marked dependence of cancer cells on the activity of the Akt/mTOR pathway makes it a reasonable target for drug research [3].

A new anti-cancer drug, N-[(2-hydroxy-5-nitrophenyl)amino]carbonothioyl-3,5dimethylbenzamide (DM-PIT-1), that targets the above pathway was recently reported [4]. It is a small molecule inhibitor of PI3K/PH binding that inhibits tumor growth. Because this drug lacks sufficient stability in biological fluids, a second generation of analogs of DMPIT-1 with improved stability was developed [5, 6]. However, since these drugs are highly water insoluble, nanocarriers have been developed that improve drug solubility and delivery $[5,7,8]$.

We have used two of the widely used lipid-based nanocarriers, lipid-core micelles and liposomes, for encapsulation and delivery of NCL-240. The nanometer size range of these delivery systems makes them appropriate for passive targeting of drugs to tumors by utilizing the well-known enhanced permeability and retention (EPR) effect [9]. The efficacy of both micellar and liposomal formulations can be further improved by active targeting. Surface modifications with ligands specific for receptors over-expressed in cancers, or antibodies specific to tumor surface antigens, can enhance the accumulation of these nanocarriers and their encapsulated drug in a tumor. Among these, cancer cells are known to overexpress transferrin (Tf) receptors many-fold compared to normal cells [10,11]. Hence, targeting the tumors with Tf-conjugated drug delivery systems represents a logical approach for drug delivery. The micelles and liposomes can be surface-modified with the Tf ligand via p-nitrophenylcarbonyl (pNP) groups [12, 13].

While multiple in vitro studies have been carried out to study the anti-cancer effects of drugs and drug delivery systems, most incorporate only monolayer cell culture models. Such monolayer cell cultures are a good reference point for the study of drug effects on cellular mechanisms of the cancer cell. However, the tumor microenvironment also plays a major role in deciding the fate of the treatments, and promising leads identified through in vitro analyses have often failed in in vivo trials [14]. 
Spheroids, as 3-dimensional cell cultures, can be used to more closely mimic the solid in vivo tumor. In addition to the effects on cellular mechanisms, application of spheroid technology can be useful in understanding the fate of the drugs or drug delivery systems in a simulated tumor microenvironment [15-18]. The 3-dimensional structures in spheroid cell cultures develop multiple cell-to-cell contacts and many other characteristics of in vivo tumor tissues. Spheroids allow for the development of a tumor-like microenvironment where they exhibit cellular and microenvironmental aspects of tumor growth, including a diffusion gradient of nutrients and oxygen, similar ratios of gene expression, accumulation of metabolites within the tissue, development of a necrotic core, and difference in proliferation of cells at periphery compared to the core $[19,20]$. NCI/ADR-RES spheroids expressing Tf receptors were developed in a previous study [21].

In this paper, we report on the use of lipid-core micelles and liposomes as nanocarriers for NCL-240 (an analog of DM-PIT-1) using NCI/ADR-RES cells cultured as spheroids.

\section{MATERIALS AND METHODS}

\section{Materials}

1,2-Distearoyl-sn-glycero-3-phosphoethanolamine-N-[methoxy(polyethylene glycol)-2000] ( $\left.\mathrm{PEG}_{2000}-\mathrm{PE}\right)$ (Corden Pharma Switzerland LLC, Liestal, Switzerland), and 1,2- dioleoylsn-glycero-3-phosphoethanolamine (DOPE) were obtained from Avanti Polar Lipids Inc. (Alabaster, AL). pNP-PEG 3400 -pNP was obtained from Laysan Bio (Arab, AL). The commercial lipids were used directly without further purification. The DM-PIT-1, analog NCL-240, was synthesized at the National Chemical Laboratory (Pune, India). Human holoTf used for surface modification of the micelles was obtained from Sigma Aldrich (St. Louis, $\mathrm{MO})$. Bicinchoninic acid assay $\left(\mathrm{BCA}^{\mathrm{TM}}\right)$ kits for determining protein concentrations were purchased from Thermo Scientific (Rockford, IL). 1,2-dimyristoyl-sn-glycero-3phosphoethanolamine-N-(lissamine rhodamine B sulfonyl) (Rh-PE) was purchased from Avanti Polar Lipids, Inc. (Alabaster, AL).

Cell culture

NCI/ADR-RES (National Cancer Institute, Frederick, MD) cells were cultured in Dulbecco's modification of Eagles Medium (DMEM; CellGro, Kansas City, MO). The media was supplemented with $10 \%$ Fetal Bovine Serum (FBS) and a $1 \%$ antibiotic solution $(10,000 \mathrm{IU} / \mathrm{ml}$ penicillin, $10,000 \mu \mathrm{g} / \mathrm{ml}$ streptomycin, and $25 \mathrm{ug} / \mathrm{ml}$ amphotericin B) to obtain serum-containing complete media. Tissue-Tek® O.C.T. compound (Sakura Finetek USA, Inc., CA, USA), Protocol ${ }^{\circledR}$ Harris' hematoxylin (Fisher Scientific, USA) and Eosin Y (Sigma Aldrich, St. Louis, MO) were purchased for H\&E staining. The CellTiter-Glo® Luminescence assay was purchased from Promega, Madison, WI, and the Accumax ${ }^{\circledR}$ Cell Dissociation solution was from Innovative Cell Technologies, San Diego, CA. Hoechst 33342 was obtained from Molecular Probes, Inc., Eugene, OR.

\section{Methods}

i. Synthesis of pNP-PEG $3400-P E-p N P-P_{3400}-\mathrm{PE}$ was synthesized based on a previously developed protocol [13]. Briefly, pNP- $\mathrm{PEG}_{3400}-\mathrm{pNP}$ in anhydrous chloroform, 
DOPE and triethylamine (TEA) were added to a $50 \mathrm{ml}$ pear shaped flask. The reaction was allowed to occur at room temperature (RT) overnight under nitrogen with constant stirring. The organic solvents were removed by rotary evaporation. The formed film was freeze dried for at least $4 \mathrm{~h}$. The film was then rehydrated using $1.0 \mathrm{mM} \mathrm{HCl}$ and separated on a sepharose column. The elution samples were collected and examined using thin-layer chromatography (TLC) to determine the fractions in which the $\mathrm{PNP}^{-\mathrm{PEG}_{3400}-\mathrm{PE} \text { product }}$ was eluted. The fractions that tested positive on the TLC were pooled, lyophilized, weighed and reconstituted in anhydrous chloroform to make the required stock solutions. The stock was stored at $-80^{\circ} \mathrm{C}$.

ii. Drug-loaded lipid-core micelles-Drug-loaded lipid-core micelles were prepared using a thin film hydration technique $[8,22]$. Briefly, films of $\mathrm{PEG}_{2000}-\mathrm{PE}$ with or without $6 \% \mathrm{NCL}-240(\mathrm{w} / \mathrm{w})$ were prepared by rotary evaporation in a round bottom flask and freeze dried using a Freezone 4.5 Freeze Dry System (Labconco, Kansas City, MO). The lipid films were then rehydrated with $1 \times$ phosphate buffered saline (PBS) pH 7.4 to achieve a $15 \mathrm{mg} / \mathrm{ml}$ total lipid concentration followed by vortexing for 5-10 minutes. The resultant micelles were centrifuged at 13,000 rpm for 5 minutes and sterile filtered through a $0.2 \mu \mathrm{m}$ polyethersulfone (PES) filter (Nalgene, Rochester, NY).

To analyze the amount of the drug loading in micelles, a reverse phase Xbridge C18 $(2.1 \mathrm{~cm}$ $\times 250 \mathrm{~cm}$ ) column (Waters Corporation, Milford, MA) was used on a Hitachi Elite LaChrom HPLC with autosampler (Pleasanton, CA). The mobile phase consisted of an isocratic mixture of 80:20 acetonitrile:water at a $1 \mathrm{ml} / \mathrm{min}$ flow rate. NCL-240 was detected as a peak in the absorption spectrum at a wavelength of $300 \mathrm{~nm}$ at a retention time of 5 to 5.5 minutes. The concentration of NCL-240 was obtained by measuring the area under the curve of the chromatogram and extrapolating it via a standard curve of NCL-240.

iii. Preparation of transferrin-conjugated micelles (Tf-micelles)-Tf-micelles were also prepared by a thin film hydration technique [22]. Briefly, $\mathrm{PEG}_{2000}-\mathrm{PE}$ in

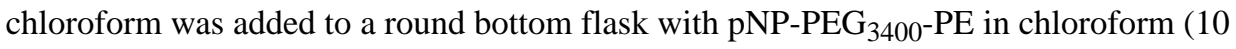
mole $\%$ of $\left.\mathrm{PEG}_{2000}-\mathrm{PE}\right)$. The organic solvents were evaporated using a rotary evaporator, and a film was formed during freeze-drying. At a molar ratio of $\mathrm{pNP}-\mathrm{PEG}_{3400} \mathrm{PE}$ to Tf of 2.5:1, a Tf stock was made in a 1:5 aqueous mixture of citrate buffered saline (CBS), $\mathrm{pH} 5.5$ and $1 \times \mathrm{PBS}, \mathrm{pH}$ 7.4. The film was rehydrated with this mixture, vortexed and the $\mathrm{pH}$ was adjusted to 8.5. The reaction continued overnight at $4{ }^{\circ} \mathrm{C}$ to allow sufficient $\mathrm{Tf}$ conjugation and complete hydrolysis of unreacted pNP groups. These micelles were dialyzed for $4 \mathrm{~h}$ at RT using a 100,000 MWCO membrane in a beaker containing 1L PBS, pH 7.4 under constant stirring, to remove the unconjugated Tf. A Micro BCA ${ }^{\mathrm{TM}}$ assay (Pierce, Rockford, IL) was carried out according to the manufacturer's protocol to measure the conjugation efficiency of the Tf to the micelles. Proton NMR spectra were run to confirm the formation of conjugates. All NMR spectra were recorded on a Varian NMR spectrometer at $500 \mathrm{MHz}$. Chemical shifts were recorded in parts per million $(\delta)$ relative to tetramethylsilane (TMS; $0.00 \mathrm{ppm}$ ) or solvent peaks as the internal reference. 
Rhodamine-PE (Rh-PE), 1 mol \%, was added along with the $\mathrm{PEG}_{2000}-\mathrm{PE}$ and pNP$\mathrm{PEG}_{3400}$-PE to prepare the films either as Rh-labeled Tf-targeted or Rh-labeled untargeted micelles.

iv. Preparation of Tf/NCL-240-micelles-As described previously [5, 23], adjacent micelles, being inherently dynamic, exchange their component monomers and form a homogenous population in solution. Hence, combinations of Tf/NCL-240-micelles were prepared by mixing appropriate amounts of NCL-240-micelles and Tf-micelles and incubation at RT for $4 \mathrm{~h}$ or overnight at $4^{\circ} \mathrm{C}$ to form a homogenous population.

\section{v. NCL-240-loaded liposomes and their characterization-ePC/Chol/DOPE/}

CHEMS liposomes with or without NCL-240 were prepared by a thin-film hydration technique [24]. The molar ratio of the lipids was kept constant throughout all experiments at $64 / 24 / 6 / 6$ respectively. Briefly, lipids in chloroform were added to round bottom flasks and the organic solvents were removed by rotary evaporation and freeze-drying to form a lipid film. The lipid film was hydrated using $1 \times$ PBS, pH 7.4 to obtain multi-lamellar vesicles (MLV) at lipid concentrations at 10 or $25 \mathrm{mg} / \mathrm{ml}$. The rehydrated liposomes were sonicated briefly and incubated at $40{ }^{\circ} \mathrm{C}$ for $15-20 \mathrm{~min}$ followed by sequential extrusion through 0.2 $\mu \mathrm{m}$ and $0.1 \mu \mathrm{m}$ polycarbonate membranes using a mini held-hand extruder (Avanti Polar Lipids Inc., Alabaster, AL). Simultaneously, a 2 mol\% PEG $_{2000}$-PE film was prepared. This film was hydrated with the liposomal solution and incubated overnight in a shaker at $37{ }^{\circ} \mathrm{C}$ to generate PEGylated liposomes by post-insertion. The liposomes were sterile filtered through $0.2 \mu \mathrm{m}$ polyethersulfone or nylon membrane filters.

vi. Transferrin-conjugated liposomes -1 mol\% $\mathrm{pNP}-\mathrm{PEG}_{3400}-\mathrm{PE}$ film was prepared independently of NCL-240 liposomes. The film was rehydrated with PBS containing Tf, adjusted to $\mathrm{pH}$ 8.0. The molar ratio of $\mathrm{pNP}-\mathrm{PEG}_{3400}-\mathrm{PE}$ :Tf was 2.5:1 and the conjugation reaction with Tf was carried out overnight. The final lipid concentration was $15 \mathrm{mg} / \mathrm{ml}$ for the resulting Tf-pNP-PEG $3400-\mathrm{PE}$ micelles which were then dialyzed against an excess of $1 \times$ PBS, pH 7.4 for $4 \mathrm{~h}$. The Tf-conjugation efficiency was measured using the Micro BCA ${ }^{\mathrm{TM}}$ assay. The Tf-PEG $3400-\mathrm{PE}$ was inserted into the bilayer of NCL-240 liposomes by overnight mixing in a shaker at $37^{\circ} \mathrm{C}$.

For cell association studies, $0.5 \mathrm{~mol} \% \mathrm{Rh}-\mathrm{PE}$, was added along with the lipid films to generate either Rh-labeled untargeted or Tf-targeted liposomes.

vii. Characterization of delivery systems-The micelles and liposomes were characterized for their size, using a Coulter N4 Plus (Beckman Coulter, Pasadena, CA) and zeta-potential using a Zetaplus (Brookhaven Instruments Corporation, Holtsville, NY). The size and zeta potential were measured according to the manufacturer's protocols with samples diluted appropriately in distilled water.

viii. Tf-targeted delivery systems' association with cells-The association of Tfmicelles and Tf-liposomes with NCI/ADR-RES cells was studied using fluorescenceassisted cell sorting (FACS) (BD FACS Caliber flow cytometer, San Jose, CA). NCI/ADRRES cells were seeded in 12 well plates at a concentration of $1 \times 10^{5}$ cells/well and 
incubated overnight at $37{ }^{\circ} \mathrm{C}$ and $5 \% \mathrm{CO}_{2}$. After $24 \mathrm{~h}$ incubation, the media was replaced with fresh media and the cells were treated with $1 \mathrm{~mol} \% \mathrm{Rh}-\mathrm{PE}$ plain micelles or Tfconjugated Rh-PE micelles (lipid at $15 \mathrm{mg} / \mathrm{ml}$ ) at 0.67 or $1.34 \mathrm{~mol} \%$ Tf ligand.

Alternatively, the cells were also treated with freshly prepared Rh-PE plain liposomes or Tfconjugated Rh-PE liposomes (lipid at $15 \mathrm{mg} / \mathrm{ml}$ ) at $0.5,1.0$ or $2.0 \mathrm{~mol} \%$ Tf ligand. The amount of Rh-PE was kept constant for all treatments. After treatment with the formulations for $2 \mathrm{~h}$, the media was removed, and the cells were harvested with trypsin-EDTA. The cell suspension was washed thrice with cold PBS, suspended in 250-300 $\mu \mathrm{l} \mathrm{PBS}$ and analyzed immediately by FACS.

ix. Spheroid cell culture-NCI/ADR-RES cells, maintained in an incubator at $37^{\circ} \mathrm{C}$ with $5 \% \mathrm{CO}_{2}$ in $\mathrm{T} 150$ flasks until 70-80\% confluence, were trypsinized, counted and suspended in media for spheroid culture. Spheroids were developed by the non-adhesive liquid overlay method described by Friedrich, et al. [25] and optimized as outlined in [26]. Each well of a 96-well flat bottom, clear, tissue culture plate was coated with $50 \mu$ of sterile $1.5 \%$ agar (Fisher Scientific, New Haven, NJ) in serum-free media. The plates were cooled for 45 minutes to form a non-adhesive coating with a concave meniscus. Cells, $1 \times 10^{4}$ in $100 \mu \mathrm{l}$, were seeded in wells of the prepared plates and centrifuged at $1500 \mathrm{~g}$ for $15 \mathrm{~min}$ using an Eppendorf 5804 R centrifuge (Hamburg, Germany). The media in the 96-well plates was replaced on alternate days with minimum spheroid disturbance. The compactness of spheroids was analyzed daily by visual inspection with a Nikon ECLIPSE E400 microscope and by light mechanical agitation using pipettes. SPOT 5.0 software was used to measure the size of the spheroids.

\section{x. Hematoxylin \& eosin staining of NCI/ADR-RES spheroid sections-Using} large orifice pipette tips, whole spheroids were removed from the wells and washed gently with excess of PBS. The spheroids were transferred to a micro-centrifuge tube containing Tissue-Tek® O.C.T. compound. This suspension was transferred onto the slicing platform in a Thermo Scientific ${ }^{\mathrm{TM}}$ Microm HM550 cryostat (Fisher Scientific, USA). The spheroids were sectioned at a thickness of $8 \mu \mathrm{m}$, mounted on a slide and allowed to dry at RT before further processing. Sections taken at depths between 40 and $64 \mu \mathrm{m}$ were selected for $\mathrm{H} \& \mathrm{E}$ staining. The spheroids sections were cleared using xylene followed by rehydration with 3 decreasing concentrations $(100 \%, 85 \%$ and $70 \%)$ of ethanol. The sections were washed with deionized water and stained with Protocol@ Harris' hematoxylin for seven minutes. The stained sections were washed with water and decolorized with acid alcohol. They were then treated with a bluing agent (1.36\% lithium carbonate) and immediately washed with water. It was followed by eosin Y staining for $30 \mathrm{sec}$ and dehydration with 3 increasing concentrations of ethanol $(80 \%, 90 \%$ and $100 \%)$. The slides were viewed under the Nikon ECLIPSE E400 microscope, and images were taken with SPOT 5.0 software.

xi. Drug delivery system penetration of spheroids-NCI/ADR-RES spheroids were treated with Tf-targeted ( 0.67 or $1.34 \mathrm{~mol} \%$ Tf) and untargeted Rh-labeled micelles or with Tf-targeted $(0.5,1.0$ or $2.0 \mathrm{~mol} \% \mathrm{Tf})$ and untargeted Rh-labeled liposomes. To study penetration of the delivery systems, the spheroids were collected after $2 \mathrm{~h}$ incubation with $\mathrm{Rh}$-labeled formulations and washed with excess of PBS to remove unassociated 
formulations. They were transferred into 8 well optical chamber slides (Lab-Tek Chamber Slide System, Fisher Scientific) with $200 \mu \mathrm{l}$ of PBS and analyzed immediately on a Zeiss LSM 700 confocal microscope with a 10× objective and 575-595 nm (Ex-Em) of rhodamine B with a $555 \mathrm{~nm}$ laser. Z-stack images were obtained at fixed intervals of $11 \mu \mathrm{m}$ from the periphery into the spheroid. Using ImageJ software, an area was selected within each Zstack spheroid image to quantify the fluorescence intensity of rhodamine, which correlated with the spheroid penetration by the delivery system. The corrected rhodamine fluorescence intensity was plotted against distance from the periphery for all treatments.

xii. Spheroid cell viability-After the cultured NCI/ADR-RES spheroids reached their full compactness (day 4/5), they were treated with test formulations in serum-free media. The spheroids were treated for $4 \mathrm{~h}$ with drug-free micelles (empty micelles), untargeted NCL-240-micelles or Tf-NCL-240-micelles. The spheroids were likewise treated for $2 \mathrm{~h}$ with untargeted NCL-240-liposomes or Tf-NCL-240-liposomes. Following the treatments, serum-free media was replaced with serum-containing complete media for a further $24 \mathrm{~h}$.

A CellTiter-Glo ${ }^{\circledR}$ Luminescence assay was performed to evaluate the overall viability of the spheroids. Briefly, the spheroids in replicate groups of 5 were collected into microcentrifuge tubes. First, the spheroid cells were dissociated with $200 \mu \mathrm{l}$ of Accumax ${ }^{\circledR}$ Cell Dissociation solution at $37^{\circ} \mathrm{C}$ with constant stirring for $10-15 \mathrm{~min}$, followed by multiple pipetting. An equal volume of FBS was added to stop the reaction. The tubes were centrifuged at 2,000 rpm for $5 \mathrm{~min}$ to form cell pellets and resuspended in a total of $200 \mu \mathrm{l}$ of a media-CellTiter-Glo ${ }^{\circledR}$ mixture (1:1). The cells were lysed by vortexing to form a clear solution. A suspension from each replicate $(100 \mu \mathrm{l})$ was added to a $96-$ well black plate with a clear bottom and luminescence was measured with a Biotek Synergy HT plate reader (Biotek, Winooski, VT) after $10 \mathrm{~min}$. The cell viability was calculated relative to the luminescence of the untreated spheroids (controls) and expressed as cell viability (\%), estimated using the formula:

Cell viability $(\%)=($ absorbance of treated cells/absorbance of untreated cells $) * 100$.

xiii. Statistical analysis-The data was generated in triplicate samples for statistical analysis. The results are reported as mean \pm standard deviation. For statistical analysis, a one-way ANOVA was performed followed by Tukey's multiple comparison tests.

\section{RESULTS}

\section{Polymeric micelle characterization}

$\mathrm{PEG}_{2000}-\mathrm{PE}$ micelles were characterized for their size and zeta potential (Table 1). The encapsulation of NCL-240 into micelles did not change the size and zeta potential of the $\mathrm{PEG}_{2000}$-PE micelles. As expected, the surface conjugation with $\mathrm{Tf}(80 \mathrm{KDa})$ increased the size of the micelles from $17.8 \pm 1.1 \mathrm{~nm}$ to $38.2 \pm 5.2 \mathrm{~nm}$ and the zeta potential to -31.4 $\pm 0.9 \mathrm{mV}$ with $0.67 \mathrm{~mol} \% \mathrm{Tf}$. An increase in Tf concentration led to a further increase in the size of the micelles.

Pharm Res. Author manuscript; available in PMC 2017 October 01. 
The encapsulation efficiency of the NCL-240 drug in the micelles was calculated using reverse phase HPLC [5]. Addition of $6 \% \mathrm{w} / \mathrm{w}$ of NCL-240 to films of $\mathrm{PEG}_{2000}-\mathrm{PE}$ resulted in a loading efficiency of $92.1 \pm 4.4 \%(n=3)$. The total lipid concentration in the system was $15 \mathrm{mg} / \mathrm{ml}$.

A Micro BCA ${ }^{\mathrm{TM}}$ assay was performed on Tf-micelles to estimate the Tf-conjugation yield. The Tf-micelles were measured against a bovine serum albumin standard provided with the kit. With an initial loading of $11.5 \mathrm{mg}$ transferrin per $11.6 \mathrm{mg}$ of total lipids, the conjugation efficiency was $32.8 \pm 3.2 \%$ ( $n=3$ ). ${ }^{1} \mathrm{H}$ NMR confirmed the formation of the Tf-PEG ${ }_{3400}-\mathrm{PE}$ formation (Supplementary Figure 1).

Subsequently, the combination formulations were prepared by co-incubation of NCL-240micelles and Tf-micelles for at least $4 \mathrm{~h}$ at RT. The lipid mixing and intrinsic dynamic characteristics of micelles resulted in formation of an uniform population of Tf/NCL-240micelles [23].

\section{Characterization of NCL-240 liposomes}

The liposomes were tested for their ability to encapsulate NCL-240 using HPLC. The amount of NCL-240 encapsulated using a $4 \% \mathrm{w} / \mathrm{w}$ initial loading in $10 \mathrm{mg} / \mathrm{ml}$ liposomes was $315 \mu \mathrm{g} / \mathrm{ml}$ (encapsulation efficiency $=95.2 \pm 3.8 \%$ ). As indicated in Table 1, the plain liposomes had a size of $144.2 \pm 3.7 \mathrm{~nm}$ and a negative zeta potential at $-42.3 \pm 6.6 \mathrm{mV}$. NCL-240-loaded liposomes had similar size and zeta potential as the plain liposomes. Surface modification of the liposomes with Tf increased the liposome size by 24 to $51 \%$.

\section{Tf-conjugated delivery system's association with cells}

NCI/ADR-RES cells were incubated in 12 well plates with untargeted and Tf-targeted Rhlabeled micelles or untargeted and Tf-conjugated Rh-labeled liposomes for $2 \mathrm{~h}$ followed by harvesting and FACS analysis. The mean fluorescence units of Rh-labeled formulations are presented in Figure 1. A clear increase in the association of micelles with NCI/ADR-RES cells after surface modification with Tf occurred at both Tf concentrations (Figure 1 (a)). A highly significant increase in association of the Tf-conjugated liposomes over the untargeted liposomes was observed using 0.5, 1.0 and $2.0 \mathrm{~mol} \%$ Tf (Figure 1 (b)).

\section{Spheroid formation}

After an initial period of 2/3 days as loose cell aggregates, the NCI/ADR-RES spheroids coalesced and became compact with a diameter reduction to $\sim 550 \mu \mathrm{m}$ at day $4 / 5$ [Supplementary Figure 2; Figure 2 (a)].

\section{Hematoxylin and eosin staining of NCl/ADR-RES spheroid sections}

This assay was carried out to establish whether the NCI/ADR-RES spheroids mimicked the in vivo tumors by exhibiting characteristics including the presence of a necrotic core. $\mathrm{H} \& \mathrm{E}$ staining of spheroid sections was performed at day 7,10 and 12 of spheroid growth [Figure 2 (b)]. The nuclei and the cytoplasm were of spheroid cells exhibited distinctly different zones with varying patterns of proliferation. Day 7 spheroids revealed the presence of a 
necrotic core (indicated by arrow) and a peripheral proliferative cell layer. Typically, a gradual increase in necrosis of the core occurred.

\section{Micelle penetration of spheroids}

Targeted micelles penetrated deeper than the untargeted [Figure 3 (a)]. The corrected rhodamine fluorescence intensity vs. distance from the periphery showed a greater micelle association (as Rh pixel count) and penetration of deeper layers by micelles targeted with either 0.67 or $1.34 \mathrm{~mol} \%$ Tf-micelles [Figure 3 (b)]. Even though the size of the Tf-micelles was larger $(38-45 \mathrm{~nm})$ than the untargeted micelles $(\sim 17 \mathrm{~nm})$, the peak penetration of the Tf-micelles was at considerably greater depths. The peak presence of the untargeted micelles occurred relatively near the spheroids' periphery (at $22 \mu \mathrm{m}$ depth) and decreased with distance towards the core in contrast to the fluorescence peak intensities with 0.67 and 1.34 mol\% Tf-micelles, which were observed at $44 \mu \mathrm{m}$ and $66 \mu \mathrm{m}$, respectively.

\section{Liposome penetration of spheroids}

Confocal microscopy images depict the presence of Rh-labeled liposomes at depths from the periphery of the spheroids (Figure 4 (a)). Quantitatively, the corrected Rh fluorescence intensity (as Rh pixel count) (Figure 4 (b)) indicates enhanced targeted liposome penetration at all levels of Tf-targeting.

\section{Spheroid cell viability}

NCI/ADR-RES spheroids were treated with different combinations of targeted and untargeted NCL-240 formulations (Figure 5). The empty Tf-micelles had no effect on cell viability. A significantly greater cytotoxicity of NCL-240 was seen when delivered using Tfmicelles [Figure 5 (a)]. The increased cytotoxicity was seen with a lower dose of targeted NCL-240 $(25 \mu \mathrm{M})$ as compared to untargeted NCL-240 $(50 \mu \mathrm{M})$ micelles. Targeting the NCL-240-liposomes with transferrin also improved the cytotoxicity at $2 \mathrm{~h}$ of incubation [Figure 5 (b)]. The $2.0 \mathrm{~mol} \%$ Tf-targeted NCL-240-liposomes were significantly more toxic than the untargeted NCL-240 liposomes. Although an improvement in spheroid penetration of the $1.0 \mathrm{~mol} \%$ Tf-targeted liposomes occurred (Figure 4), an effect on cytotoxicity was not observed when compared to the untargeted liposomes.

\section{DISCUSSION}

The current study aimed to formulate two targeted lipid-based nano-delivery systems, loaded with a hydrophobic anti-cancer drug, NCL-240, and to observe their interaction with NCI/ ADR-RES cancer cells grown in a 3D spheroid model.

$\mathrm{PEG}_{2000}$-PE-based polymeric micelles were prepared by a thin-film hydration technique. The size of the NCL-240-loaded micelles was less than $20 \mathrm{~nm}$ and the zeta potential was negative. On surface-modification with transferrin, the size and the negative zeta potential of the resulting micelles increased. The increase in size of the Tf-micelles is the result of the relatively large size of $\operatorname{Tf}(80 \mathrm{kDa})$ and the more negative zeta potential is due to the net negative charge of the Tf protein. It is notable that even after conjugation with Tf, the size of the micelles remained below $50 \mathrm{~nm}$ which is considered ideal for tumor permeability-based

Pharm Res. Author manuscript; available in PMC 2017 October 01. 
drug delivery [27]. Polymeric micelles efficiently loaded more than $90 \%$ of initially-added NCL-240 within the hydrophobic core. A high loading efficiency was expected with NCL-240 because of its high lipophilicity $(\log P=5.18)$ of the drug [5].

Surface modification of the micelles with $\mathrm{Tf}$ ligand involved a conjugation step between the $\mathrm{Tf}$ and the p-nitrophenylcarbonyl (pNP) group of the pNP-PEG 3400 -PE. The success of the conjugation reactions was confirmed by the proton NMR (Supplementary data).

Liposomes prepared with ePC, CHOL, DOPE and CHEMS encapsulated $~ 95 \%$ of the added NCL-240. Again, the high lipophilicity of NCL-240 may have been responsible for the high encapsulation efficiency. The untargeted NCL-240 liposomes were homogenous in size at around $150 \mathrm{~nm}$. The size increased after surface modification with Tf to a little over $200 \mathrm{~nm}$ indicating a presence of the large $\mathrm{Tf}$ molecules on the surface of the liposomes.

An ovarian cancer cell line (NCI/ADR-RES), resistant to doxorubicin [28], was chosen to study the interactions with the Tf-based nano-delivery systems since it is known to overexpress Tf-receptors [21]. Both the delivery systems were analyzed for their association with the cells grown as monolayers. A concentration-dependent cellular association was clearly seen with targeted micelles. With liposomes, Tf-targeting induced greater cellular association. This result confirmed the suitability of NCI/ADR-RES as a model cell line for Tf-targeted micelles and liposomes.

NCI/ADR-RES cells, seeded in 96-well plates, consistently formed 90-95\% spheroids using the non-adhesive liquid overlay technique. This technique was confirmed as reproducible for spheroid generation with NCI/ADR-RES cells (Supplementary Figure 2). The spheroids became visually denser over the period of their growth. The diameter of the spheroids became smaller from the day of seeding and had a consistent and reproducible diameter of $\sim 550 \mu \mathrm{m}$ after 3 days. This size of spheroids is relevant for in vitro models since it is consistent with the larger distances between the blood capillaries in solid tumors. Compared to the normal tissues, where the distance between blood capillaries is only few cell diameters thick, solid tumors typically have cells distances averaging more than $100 \mu \mathrm{m}$ from a blood capillary [29]. The cells at the center of the NCI/ADR-RES spheroids were 150 to $200 \mu \mathrm{m}$ from the spheroid periphery compared to the intimate contact with the media when the cells are grown in a monolayer.

The H\&E stained spheroid sections revealed a distinctly differentiated core. The formation of a distinct ischemic spheroid core was clear from at least day $4 / 6$ when the spheroids had achieved a dense structure. The distinct appearance of the core also suggests that NCI/ADRRES spheroids at least somewhat represent a model consistent with in vivo tumor-like conditions and supports their appropriateness for testing chemotherapeutic formulations and drug delivery systems. The effect of the spheroid's size on the development of pathophysiological and chemical gradients is substantial. Spheroids with sizes $\sim 500 \mu \mathrm{m}$ mirror in vivo solid tumors in terms of the presence of proliferative cells closer to the blood capillaries, and of an apoptotic and necrotic core [30]. This situation leads to expression of signals in response to hypoxia and low microenvironmental $\mathrm{pH}$ that contributes to multidrug 
resistance. These signals are not observed in cells grown as monolayers at typical incubation conditions of $37^{\circ} \mathrm{C}$ and $5 \% \mathrm{CO}_{2}[31,32]$.

Micelle penetration studies into NCI/ADR-RES spheroids indicated an increased penetration with the Tf-micelles in comparison to the untargeted micelles. A slightly higher accumulation of the untargeted micelles was observed in the spheroid periphery compared to the Tf-micelles at the same depth. However, the maximum fluorescence peak intensities was observed at greater depths with the Tf-micelles compared to the untargeted micelles.

Another noteworthy point was the marked presence ( $\sim 5$ units) of Tf-micelles at depths of up to $100 \mu \mathrm{m}$ into the spheroids compared to the untargeted micelles observed only up to a depth of $66 \mu \mathrm{m}$. The Tf-targeting may have provided a 'sink effect' in which the Tf-micelles entering the spheroid are more rapidly endocytosed after interaction with Tf-receptors and so augment penetration of the targeted formulations deeper into the spheroids.

As with the untargeted micelles, the untargeted liposomes peaked more peripherally in the spheroid. Likewise, the liposomes targeted with Tf penetrated more deeply with the peak fluorescence intensities at 30 to $40 \mu \mathrm{m}$ depth. The Tf-liposomes were rapidly endocytosed by the NCI/ADR-RES cells in the spheroids as indicated by a higher rhodamine label intensity. These results mirrored the observed increased cellular association of Tf-targeted liposomes in monolayers shown by FACS. In general, both Tf-micelles and Tf-liposomes gave similar patterns of spheroid association and penetration. In both the studies, a greater penetration was observed with Tf-targeting. However, the extent of penetration was somewhat different for the two nanocarriers, with a greater micellar penetration, perhaps related to the difference in size of the nanocarriers.

The NCI/ADR-RES spheroids treated with the Tf-NCL-240-micelles showed that a significant increase in cell death was triggered as compared to untargeted drug-loaded micelles. The increased cytotoxicity was expected with the higher spheroid association and penetration of the Tf-micelles. Tf-targeting improved cytotoxicity of NCL-240 micelles at half the NCL-240 dose observed with untargeted micelles.

Spheroid penetration and association by targeted and untargeted drug-loaded liposomes suggested improved uptake of Tf-liposomes compared to the untargeted liposomes. However, the cytotoxicity studies indicated a significant cytotoxic effect only of $2.0 \mathrm{~mol} \%$ Tf-NCL-240-liposomes. We suggest that with the short period of treatment incubation, the $2.0 \mathrm{~mol} \%$ Tf-NCL-240-liposomes had greater internalization compared to the $1.0 \mathrm{~mol} \%$ TfNCL-240-liposomes. Overall, targeting enhanced the cytotoxicity of NCL-240.

\section{CONCLUSION}

Lipid-core micelles and liposomes were prepared to solubilize and deliver NCL-240 to spheroids of NCI/ADR-RES cancer cells. The spheroids somewhat mimic the solid tumors in vivo and thus provide an advantage for study of the effects of a targeted delivery system with the encapsulated NCL-240 in a simulated tumor micro-environment. The spheroid penetration studies indicate that targeting enhances the delivery of micelles and liposomes to 
the spheroids. The enhanced delivery of NCL-240 with such targeted delivery improves its cytotoxic effects.

\section{Supplementary Material}

Refer to Web version on PubMed Central for supplementary material.

\section{Acknowledgments}

NIH/NCI grant U54CA151881 to V.T.

\section{ABBREVIATIONS}

\begin{tabular}{ll} 
PI3K & Phosphatidylinositide 3-kinase \\
PIP2 & Phosphatidylinositol-3,4-diphosphate \\
PIP $_{3}$ & Phosphatidylinositol-3,4,5-triphosphate \\
PKB/Akt & Protein kinase B \\
PH & Pleckstrin-homology \\
PTEN & Phosphatase and tensin homologous protein \\
PE & Phosphatidylethanolamine \\
PEG & Polyethylene glycol \\
ePC & Egg phosphatidylcholine \\
CHOL & Cholesterol \\
DOPE & 1,2- dioleoyl-sn-glycero-3-phosphoethanolamine \\
CHEMS & Cholesterol hemisuccinate \\
pNP & p-nitrophenylcarbonyl \\
EPR & Enhanced permeability and retention \\
MDR & Multi-drug resistant \\
Tf & Transferrin \\
\hline
\end{tabular}

DM-PIT-1 N-[(2-hydroxy-5-nitrophenyl)amino]carbonothioyl-3,5-dimethylbenzamide NCL-240 National Chemical Laboratory (Pune, India)

\section{References}

1. Fresno Vara JA, Casado E, de Castro J, Cejas P, Belda-Iniesta C, Gonzalez-Baron M. PI3K/Akt signalling pathway and cancer. Cancer treatment reviews. 2004 Apr; 30(2):193-204. [PubMed: 15023437]

Pharm Res. Author manuscript; available in PMC 2017 October 01. 
2. Courtney KD, Corcoran RB, Engelman JA. The PI3K pathway as drug target in human cancer. Journal of clinical oncology : official journal of the American Society of Clinical Oncology. 2010 Feb 20; 28(6):1075-83. [PubMed: 20085938]

3. Liu P, Cheng H, Roberts TM, Zhao JJ. Targeting the phosphoinositide 3-kinase pathway in cancer. Nature reviews Drug discovery. 2009 Aug; 8(8):627-44. [PubMed: 19644473]

4. Miao B, Skidan I, Yang J, Lugovskoy A, Reibarkh M, Long K, et al. Small molecule inhibition of phosphatidylinositol-3,4,5-triphosphate (PIP3) binding to pleckstrin homology domains. Proceedings of the National Academy of Sciences of the United States of America. 2010 Nov 16; 107(46):20126-31. [PubMed: 21041639]

5. Riehle RD, Cornea S, Degterev A, Torchilin V. Micellar formulations of pro-apoptotic DM-PIT-1 analogs and TRAIL in vitro and in vivo. Drug delivery. $2013 \mathrm{Feb} ; 20(2): 78-85$. [PubMed: 23495715]

6. Kommagalla Y, Cornea S, Riehle R, Torchilin V, Degterev A, Ramana CV. Optimization of the anticancer activity of phosphatidylinositol-3 kinase pathway inhibitor PITENIN-1: switching a thiourea with 1,2,3-triazole. MedChemComm. 2014 Sep; 5(9):1359-63. [PubMed: 25505943]

7. Skidan I, Miao B, Thekkedath RV, Dholakia P, Degterev A, Torchilin V. In vitro cytotoxicity of novel pro-apoptotic agent DM-PIT-1 in PEG-PE-based micelles alone and in combination with TRAIL. Drug delivery. 2009 Jan; 16(1):45-51. [PubMed: 19555308]

8. Riehle R, Pattni B, Jhaveri A, Kulkarni A, Thakur G, Degterev A, et al. Combination Nanopreparations of a Novel Proapoptotic Drug - NCL-240, TRAIL and siRNA. Pharmaceutical research. 2016 Mar 7.

9. Torchilin V. Tumor delivery of macromolecular drugs based on the EPR effect. Advanced drug delivery reviews. 2011 Mar 18; 63(3):131-5. [PubMed: 20304019]

10. Danhier F, Feron O, Preat V. To exploit the tumor microenvironment: Passive and active tumor targeting of nanocarriers for anti-cancer drug delivery. Journal of controlled release : official journal of the Controlled Release Society. 2010 Dec 1; 148(2):135-46. [PubMed: 20797419]

11. Sutton D, Nasongkla N, Blanco E, Gao J. Functionalized micellar systems for cancer targeted drug delivery. Pharmaceutical research. 2007 Jun; 24(6):1029-46. [PubMed: 17385025]

12. Sawant RR, Jhaveri AM, Koshkaryev A, Qureshi F, Torchilin VP. The effect of dual ligand-targeted micelles on the delivery and efficacy of poorly soluble drug for cancer therapy. Journal of drug targeting. 2013 Aug; 21(7):630-8. [PubMed: 23594094]

13. Torchilin VP, Levchenko TS, Lukyanov AN, Khaw BA, Klibanov AL, Rammohan R, et al. pNitrophenylcarbonyl-PEG-PE-liposomes: fast and simple attachment of specific ligands, including monoclonal antibodies, to distal ends of PEG chains via p-nitrophenylcarbonyl groups. Biochimica et biophysica acta. 2001 Apr 2; 1511(2):397-411. [PubMed: 11286983]

14. Mikhail AS, Eetezadi S, Allen C. Multicellular tumor spheroids for evaluation of cytotoxicity and tumor growth inhibitory effects of nanomedicines in vitro: a comparison of docetaxel-loaded block copolymer micelles and Taxotere(R). PloS one. 2013; 8(4):e62630. [PubMed: 23626842]

15. Lin RZ, Chang HY. Recent advances in three-dimensional multicellular spheroid culture for biomedical research. Biotechnology journal. 2008 Oct; 3(9-10):1172-84. [PubMed: 18566957]

16. Breslin S, O'Driscoll L. Three-dimensional cell culture: the missing link in drug discovery. Drug discovery today. 2013 Mar; 18(5-6):240-9. [PubMed: 23073387]

17. Perche F, Patel NR, Torchilin VP. Accumulation and toxicity of antibody-targeted doxorubicinloaded PEG-PE micelles in ovarian cancer cell spheroid model. Journal of controlled release : official journal of the Controlled Release Society. 2012 Nov 28; 164(1):95-102. [PubMed: 22974689]

18. Mehta G, Hsiao AY, Ingram M, Luker GD, Takayama S. Opportunities and challenges for use of tumor spheroids as models to test drug delivery and efficacy. Journal of controlled release : official journal of the Controlled Release Society. 2012 Dec 10; 164(2):192-204. [PubMed: 22613880]

19. Takagi A, Watanabe M, Ishii Y, Morita J, Hirokawa Y, Matsuzaki T, et al. Three-dimensional cellular spheroid formation provides human prostate tumor cells with tissue-like features. Anticancer research. 2007 Jan-Feb;27(1A):45-53. [PubMed: 17352215]

20. Ota H, Miki N. Microtechnology-based three-dimensional spheroid formation. Frontiers in bioscience. 2013; 5:37-48. 
21. Sarisozen C, Abouzeid AH, Torchilin VP. The effect of co-delivery of paclitaxel and curcumin by transferrin-targeted PEG-PE-based mixed micelles on resistant ovarian cancer in 3-D spheroids and in vivo tumors. European journal of pharmaceutics and biopharmaceutics : official journal of Arbeitsgemeinschaft fur Pharmazeutische Verfahrenstechnik eV. 2014 Jul 10.

22. Sawant RR, Torchilin VP. Polymeric micelles: polyethylene glycol-phosphatidylethanolamine (PEG-PE)-based micelles as an example. Methods in molecular biology. 2010; 624:131-49. [PubMed: 20217593]

23. Reulen SW, Merkx M. Exchange kinetics of protein-functionalized micelles and liposomes studied by Forster resonance energy transfer. Bioconjugate chemistry. 2010 May 19; 21(5):860-6. [PubMed: 20397687]

24. Patel NR, Rathi A, Mongayt D, Torchilin VP. Reversal of multidrug resistance by co-delivery of tariquidar (XR9576) and paclitaxel using long-circulating liposomes. International journal of pharmaceutics. 2011 Sep 15; 416(1):296-9. [PubMed: 21703341]

25. Friedrich J, Seidel C, Ebner R, Kunz-Schughart LA. Spheroid-based drug screen: considerations and practical approach. Nature protocols. 2009; 4(3):309-24. [PubMed: 19214182]

26. Perche F, Torchilin VP. Cancer cell spheroids as a model to evaluate chemotherapy protocols. Cancer biology \& therapy. 2012 Oct; 13(12):1205-13. [PubMed: 22892843]

27. Lukyanov AN, Gao Z, Torchilin VP. Micelles from polyethylene glycol/phosphatidylethanolamine conjugates for tumor drug delivery. Journal of controlled release : official journal of the Controlled Release Society. 2003 Aug 28; 91(1-2):97-102. [PubMed: 12932641]

28. Qin M, Lee YE, Ray A, Kopelman R. Overcoming cancer multidrug resistance by codelivery of doxorubicin and verapamil with hydrogel nanoparticles. Macromolecular bioscience. 2014 Aug; 14(8):1106-15. [PubMed: 24771682]

29. Minchinton AI, Tannock IF. Drug penetration in solid tumours. Nature reviews Cancer. 2006 Aug; 6(8):583-92. [PubMed: 16862189]

30. Hirschhaeuser F, Menne H, Dittfeld C, West J, Mueller-Klieser W, Kunz-Schughart LA. Multicellular tumor spheroids: an underestimated tool is catching up again. Journal of biotechnology. 2010 Jul 1; 148(1):3-15. [PubMed: 20097238]

31. Desoize B, Gimonet D, Jardiller JC. Cell culture as spheroids: an approach to multicellular resistance. Anticancer research. 1998 Nov-Dec;18(6A):4147-58. [PubMed: 9891460]

32. Patel NR, Pattni BS, Abouzeid AH, Torchilin VP. Nanopreparations to overcome multidrug resistance in cancer. Advanced drug delivery reviews. 2013 Nov; 65(13-14):1748-62. [PubMed: 23973912] 


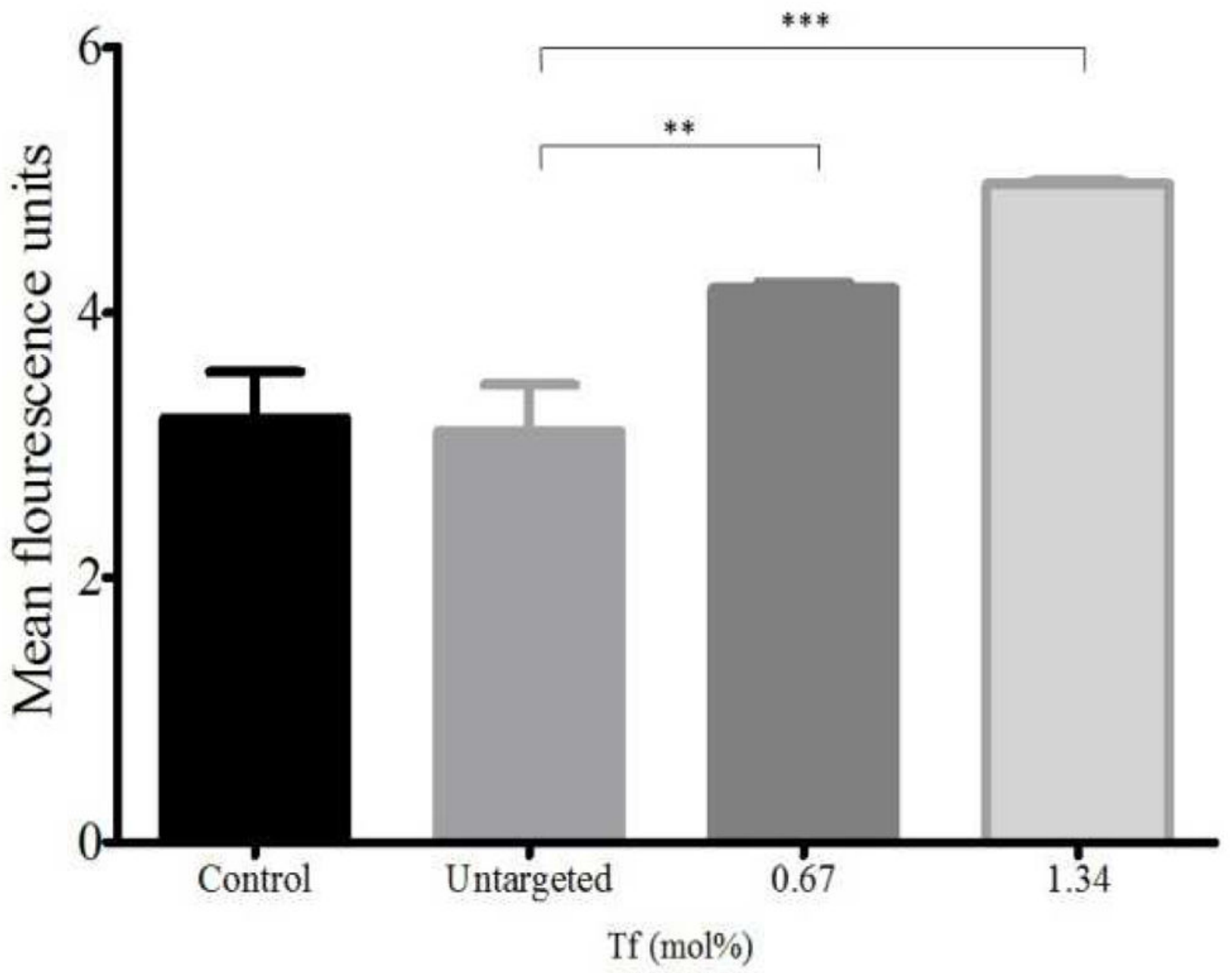

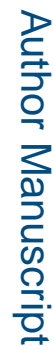

Fig 1(a) 


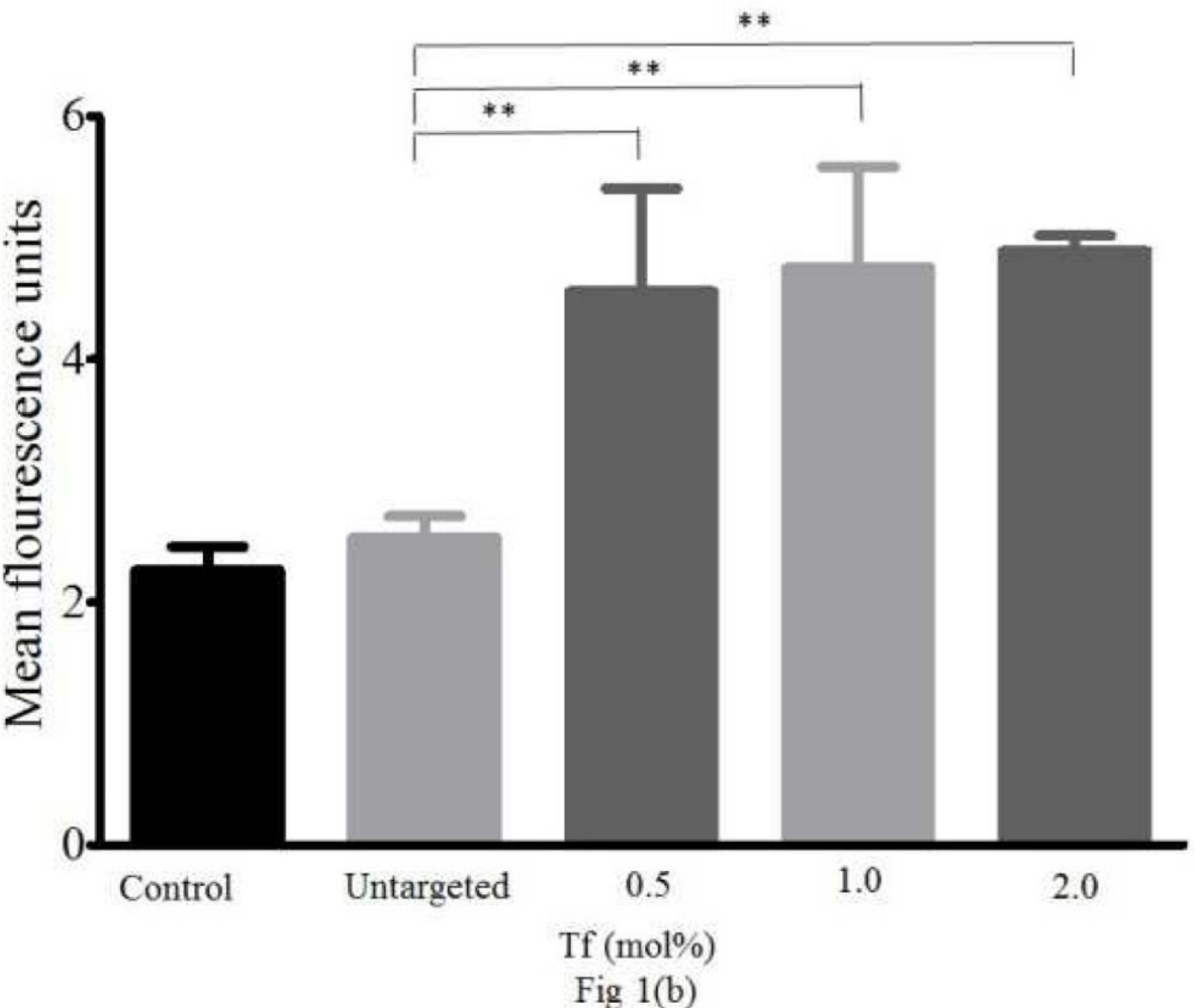

Figure 1. Cellular association of Tf-targeted (a) micelles and (b) liposomes measured using FACS (a) Association of Tf-conjugated micelles with NCI/ADR-RES. (b) Association of Tftargeted liposomes with NCI/ADR-RES cells ( $\mathrm{n}=3$, Mean \pm S.D., $* * \mathrm{p} \unlhd 0.01, * * * \mathrm{p} \unlhd$ (0.001). 


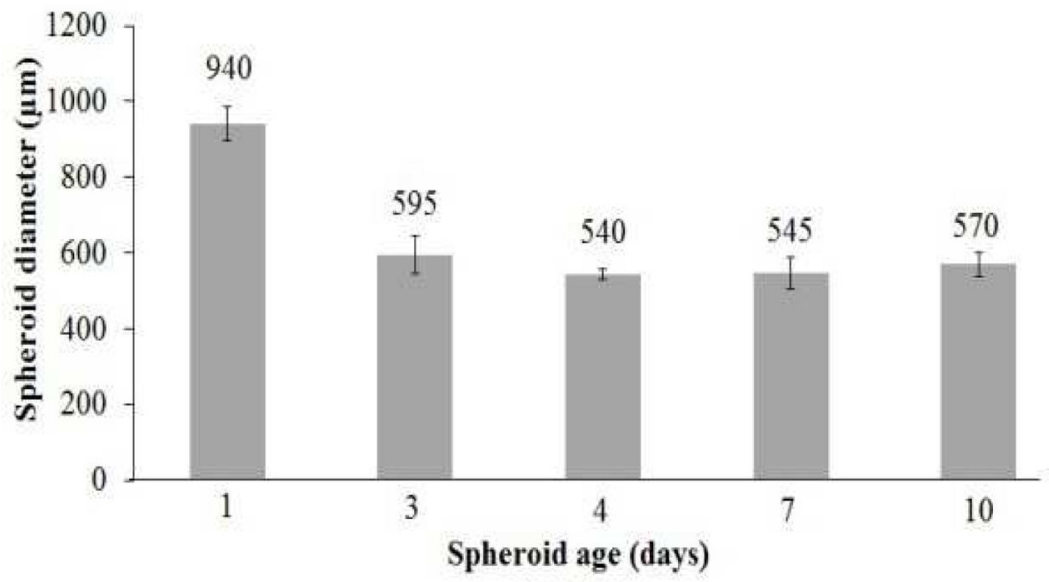

Fig 2(a)

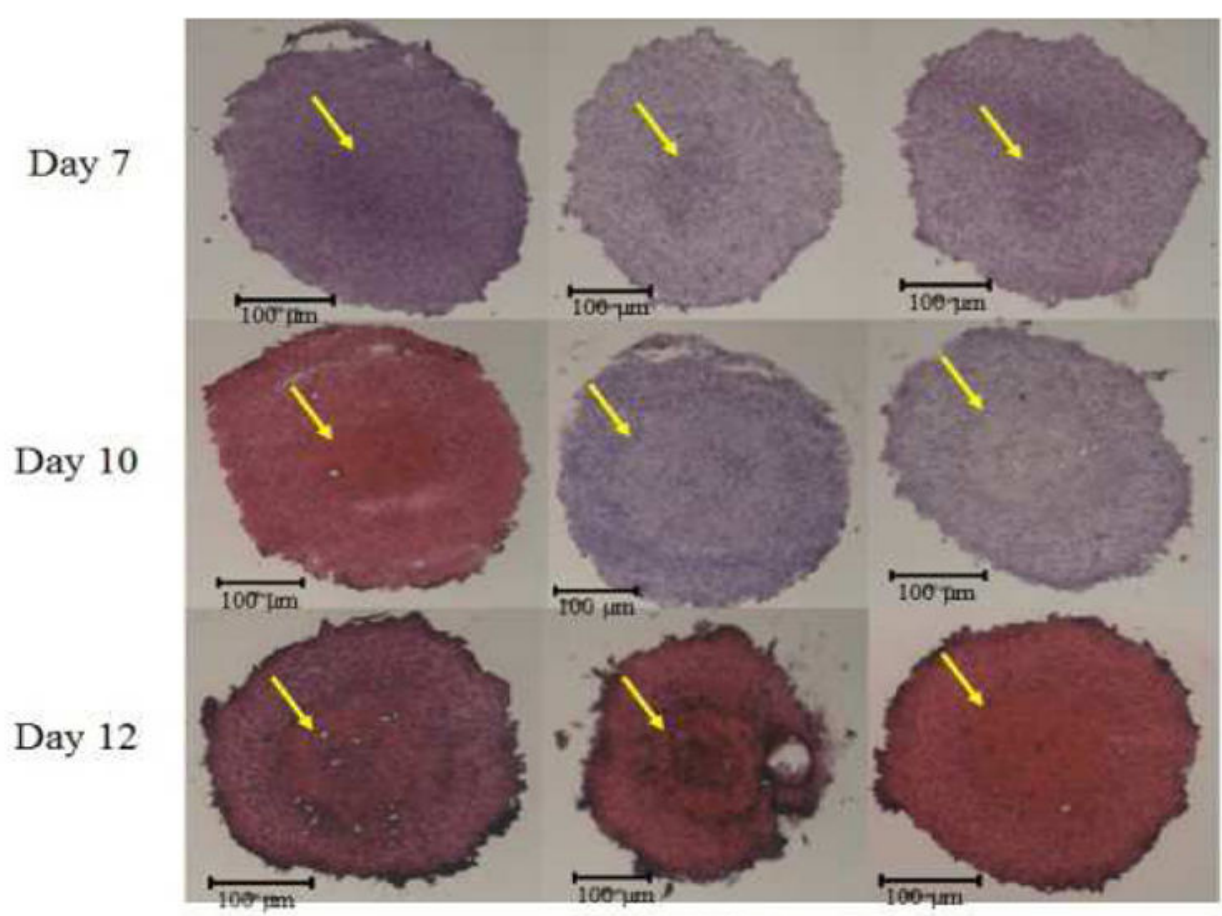

Fig 2(b)

Figure 2. NCI/ADR-RES spheroid growth

(a) Spheroid diameter with age (Mean \pm S.D., $n=3$ ) (b) H\&E stained sections of NCI/ADRRES spheroids by fluorescence microscopy. From day 7 onward, the formation of a distinctly stained core (indicated by arrow) became visible. Day 10 spheroids showed 2 distinct layers consisting of a proliferative outer shell and the necrotic inner core. By day 12, the spheroids had begun shedding cells on the periphery. 

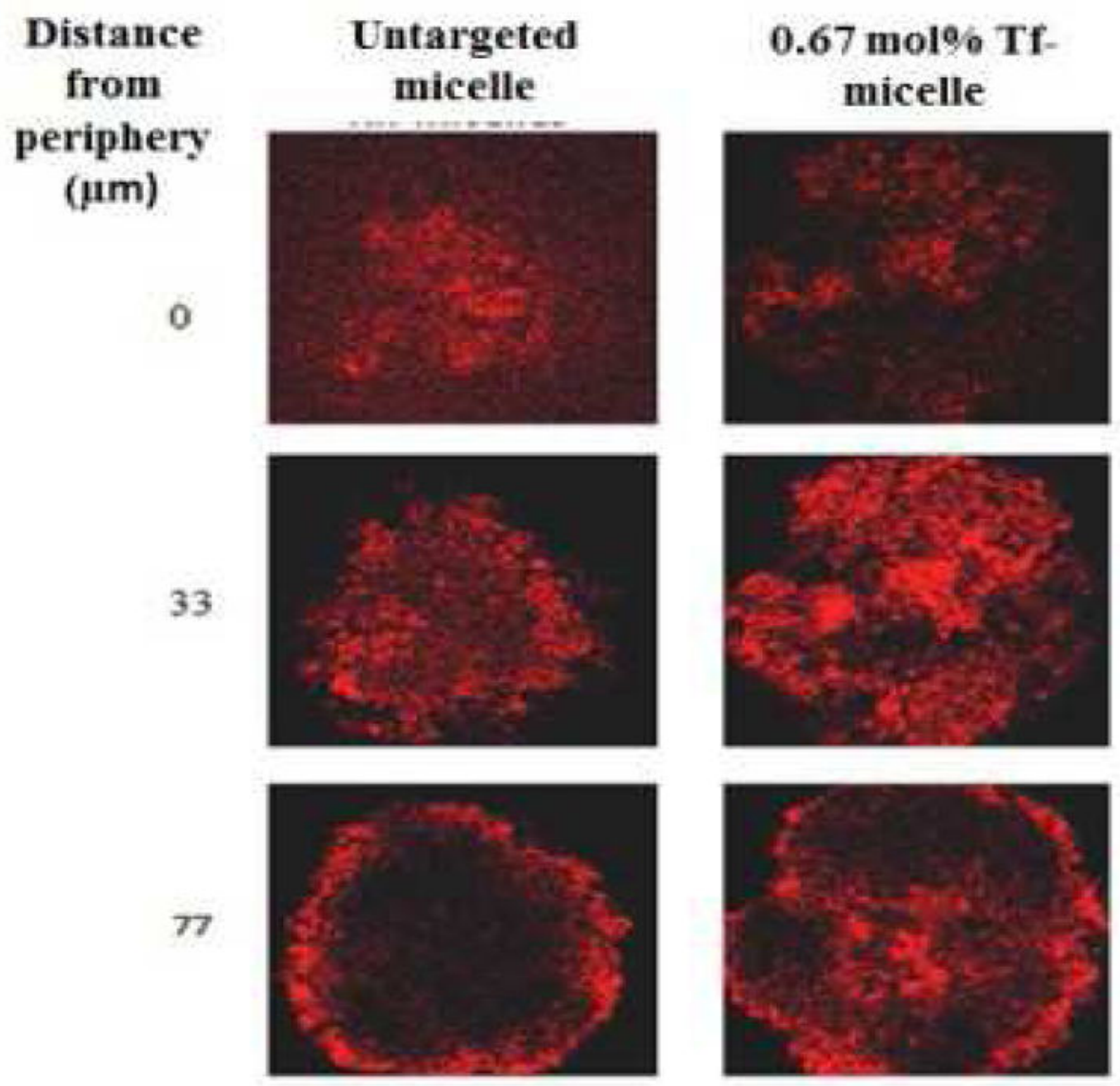

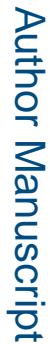

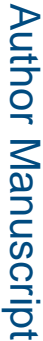

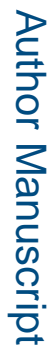

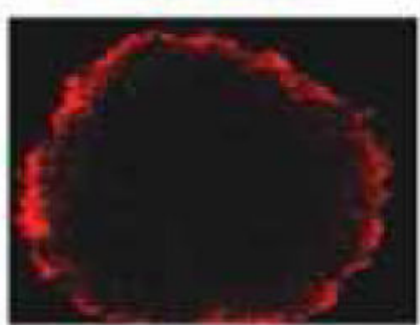

110

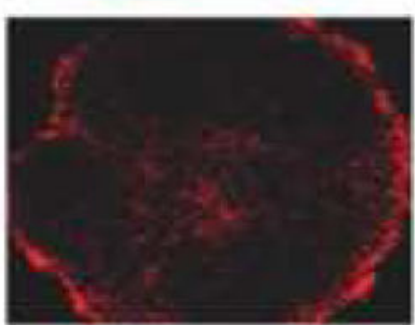

Fig 3(a)

\section{$1.34 \mathrm{~mol} \% \mathrm{Tf}-$ micelle}
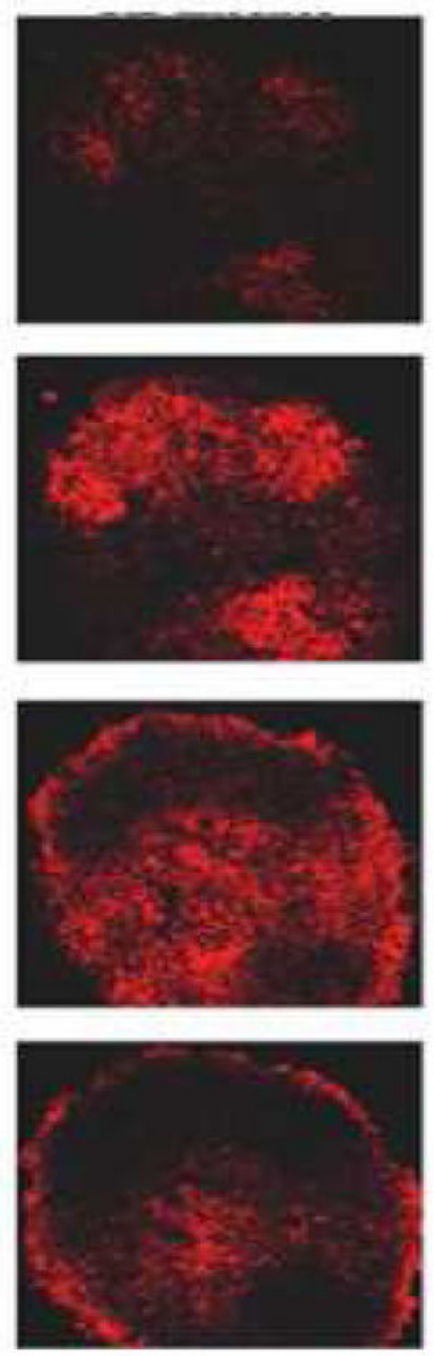


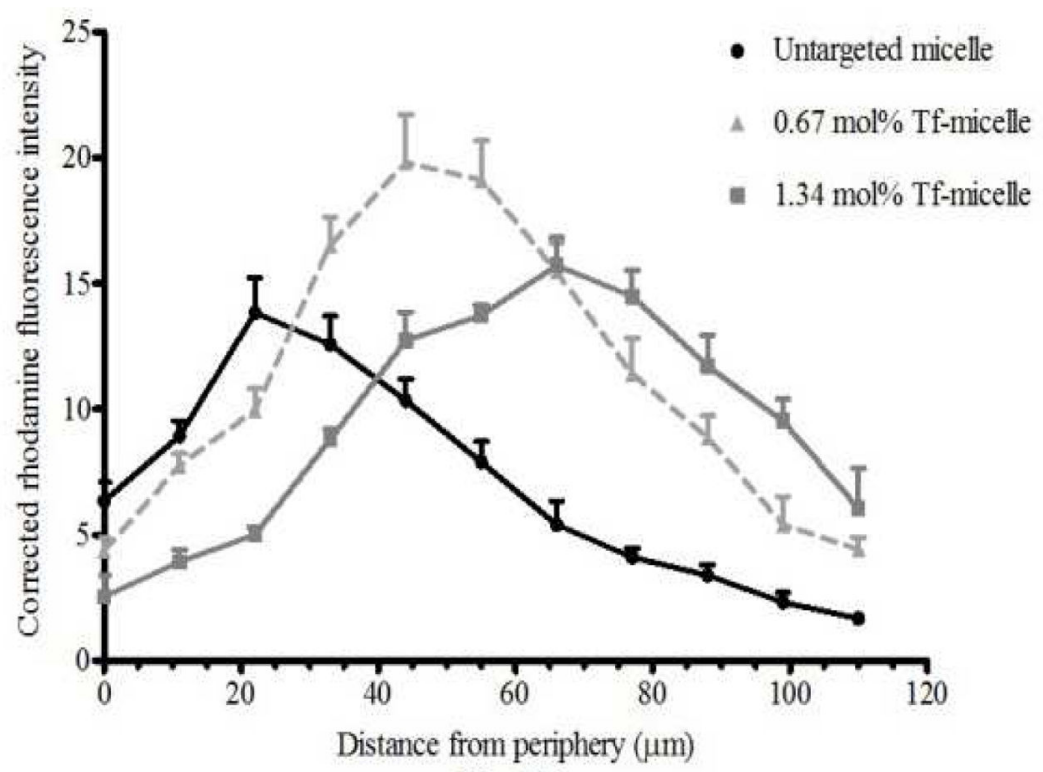

Fig 3(b)

Figure 3. Micelle penetration of spheroids

(a) Z-stack images taken by confocal microscopy showing penetration of untargeted and Tftargeted rhodamine (Rh)-labeled micelles in 4 days old NCI/ADR-RES spheroids. The images are of spheroid sections at $0,33,77$ and $110 \mu \mathrm{m}$ distance from the periphery. The red color indicates the presence of Rh-labeled micelles. The micelle association with the deeper layers of the spheroid was higher when targeted. (b) The corrected rhodamine fluorescence intensity represents the micelle association of targeted and untargeted micelles in NCI/ADRRES spheroids from the periphery to the inner layers. 


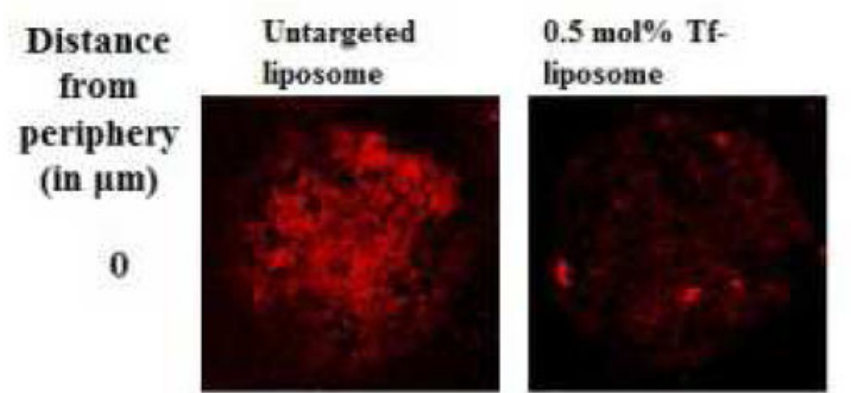

$1.0 \mathrm{~mol} \%$ Tfliposome
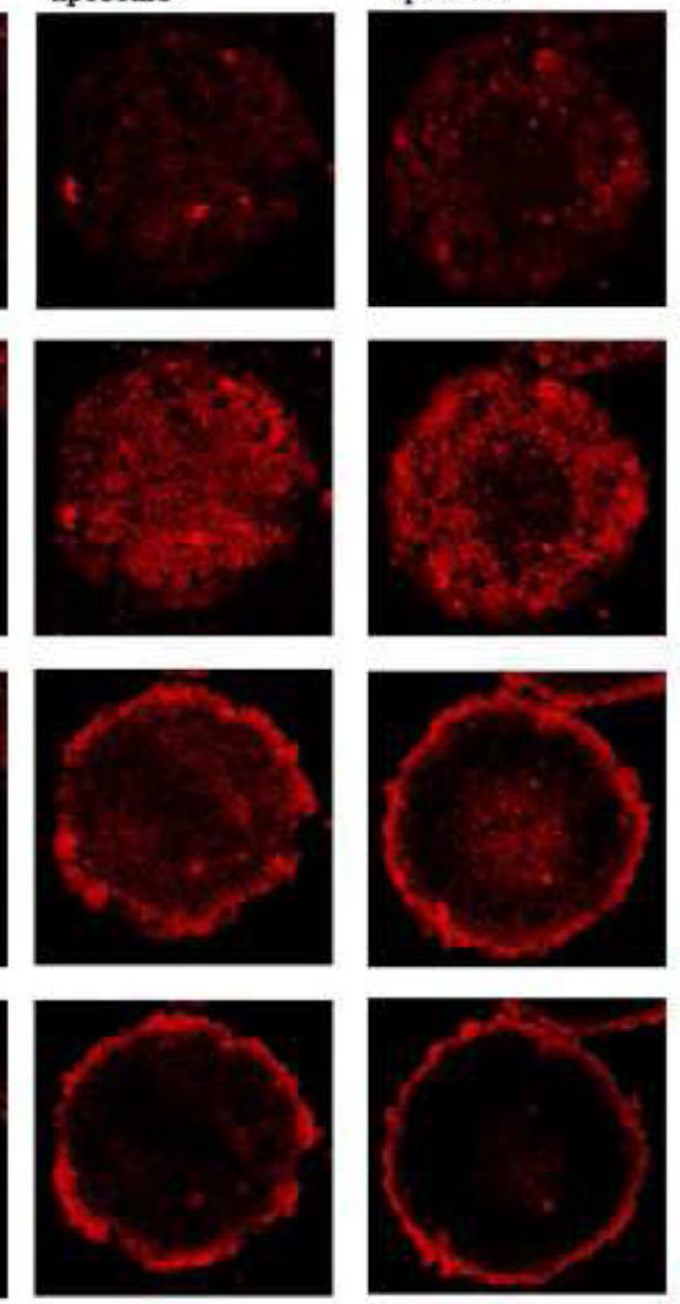

Fig 4(a)

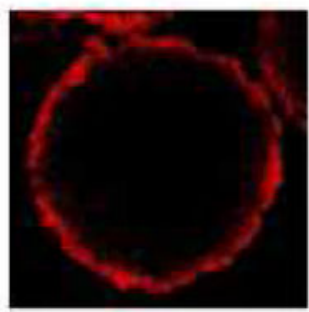

$2.0 \mathrm{~mol} \%$ Tfliposome
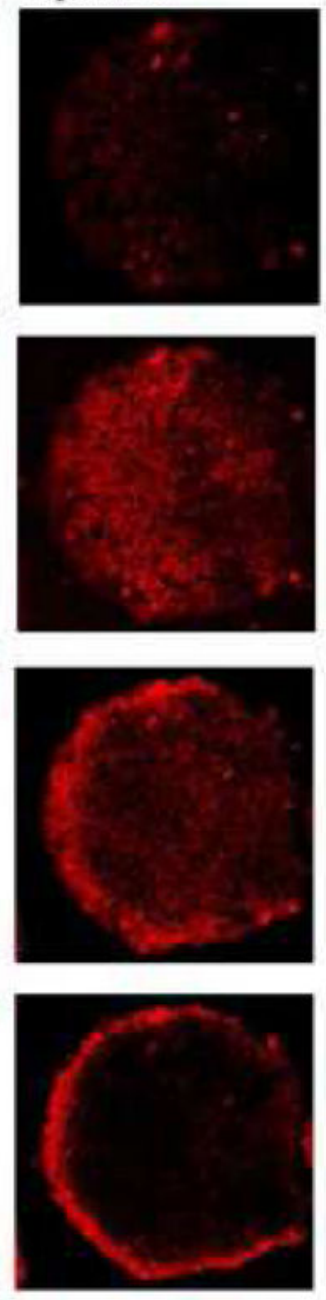


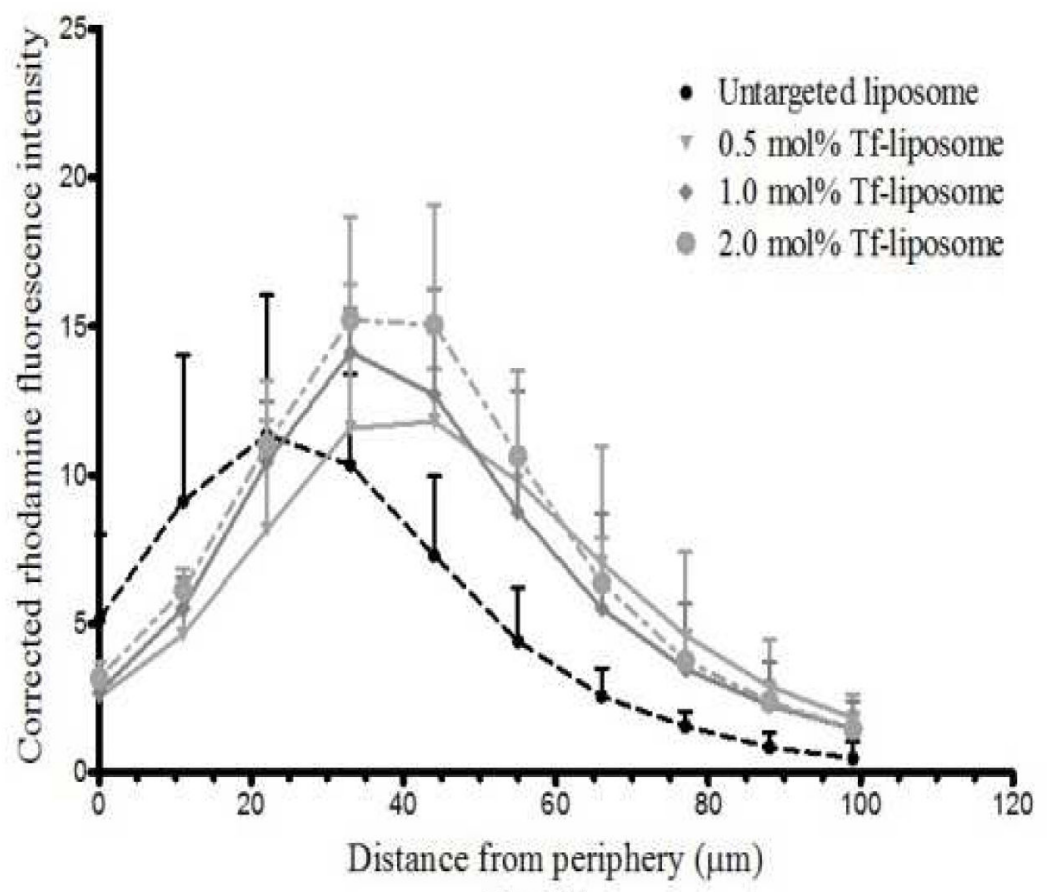

Fig 4(b)

Figure 4. Liposome penetration in spheroids

(a) Z-stack images taken by confocal microscopy show penetration of untargeted and Tftargeted rhodamine (Rh)-labeled liposomes in 4 days old NCI/ADR-RES spheroids. The images are of spheroid sections at 0,33,77 and $99 \mu \mathrm{m}$ distance from the periphery. The red color indicates the presence of Rh-labeled liposomes. The association with the deeper layers of the spheroid was higher with Tf-targeted liposomes. (b) The corrected rhodamine fluorescence intensity vs distance from the periphery quantitates the liposome association of targeted and untargeted liposomes in NCI/ADR-RES spheroids. 


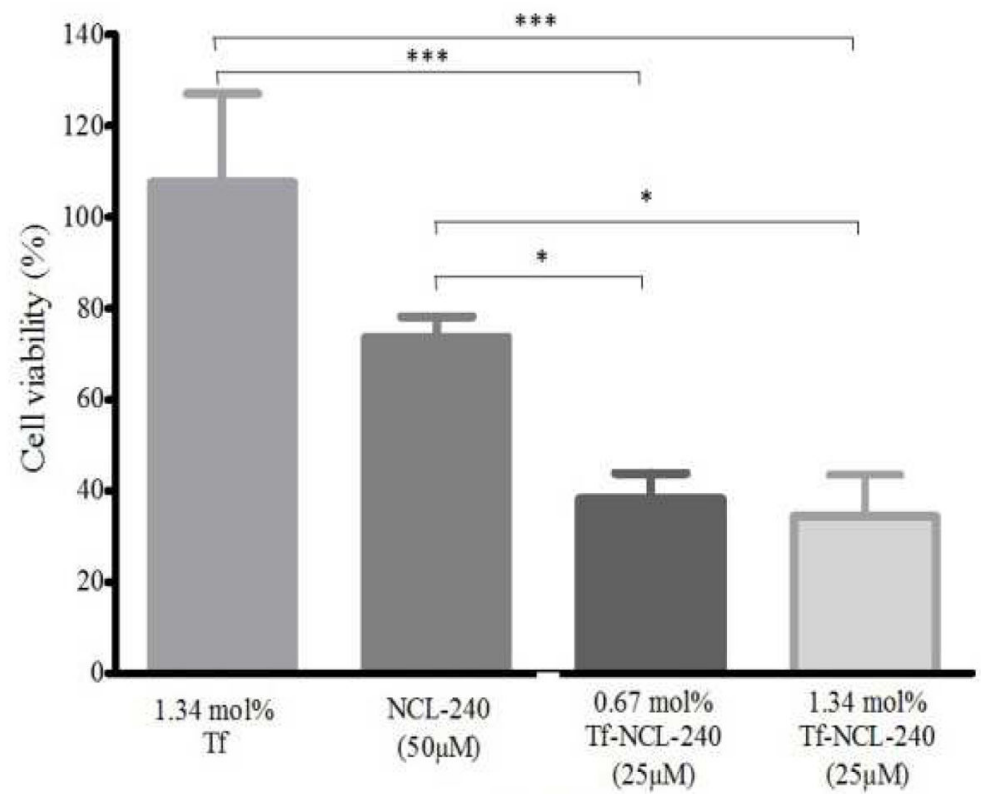

Fig 5(a) 


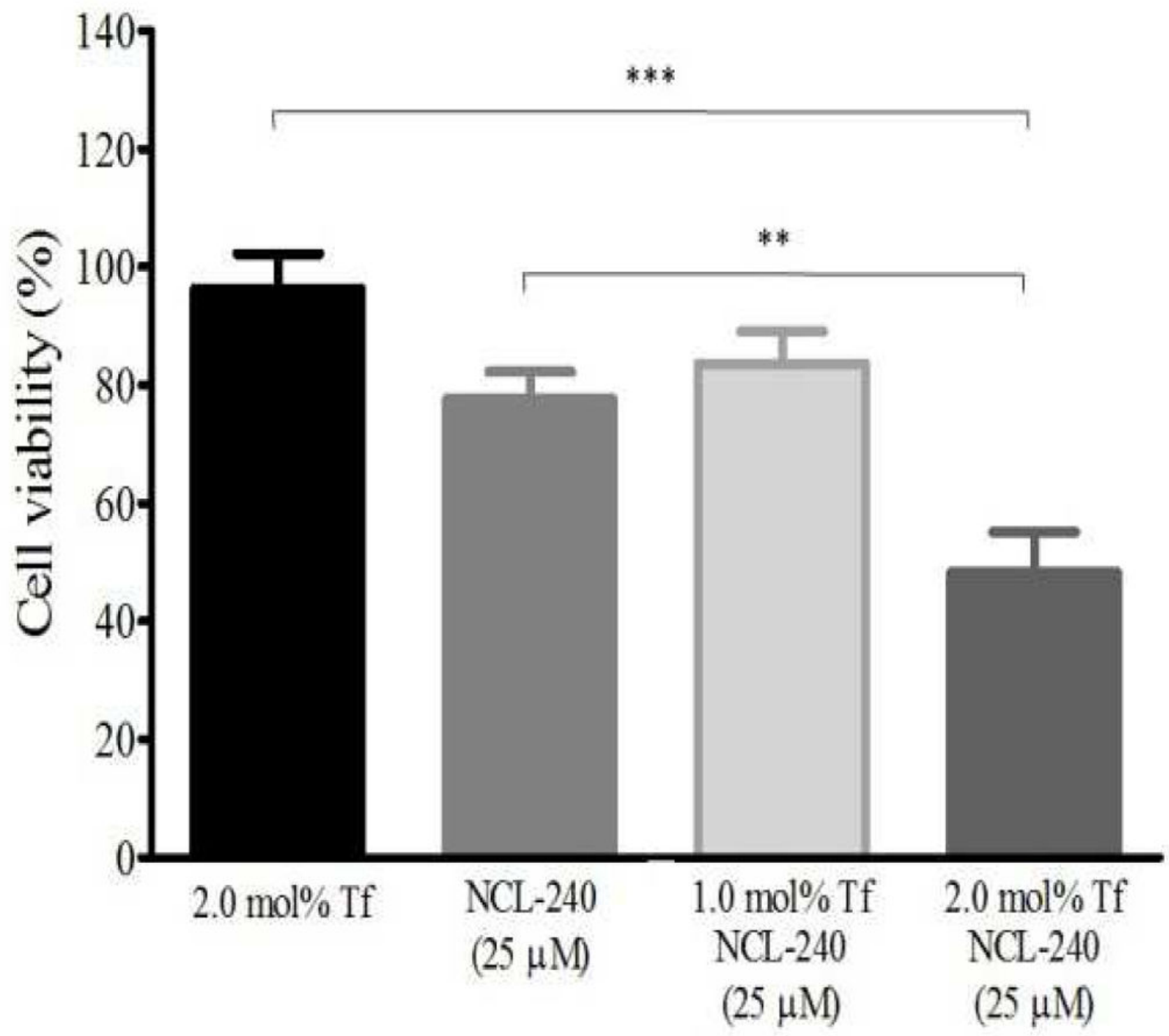

Fig 5(b)

Figure 5. Cell viability in spheroids after treatment with NCL-240-loaded (a) micelles and (b) liposomes

Cell viability in spheroids after $4 \mathrm{~h}$ treatment with NCL-240-micelles (a). With a $25 \mu \mathrm{M}$ dose of NCL-240 in Tf-targeted (0.67 or $1.34 \mathrm{~mol} \%$ ) micelles, significant cytotoxicity occurred compared to a $50 \mu \mathrm{M}$ dose of untargeted micellar NCL-240 (a). Cell viability in spheroids after $2 \mathrm{~h}$ treatment with NCL-240-liposomes (b). A $25 \mu \mathrm{M}$ dose of NCL-240 in $2.0 \mathrm{~mol} \%$ Tf-targeted in liposomes significantly increased cytotoxicity compared to untargeted NCL-240 liposomes. (n=3, Mean \pm S.D., * p $₫ 0.05$, ** p $₫ 0.01, * * * \mathrm{p} \unlhd 0.001$ ) 


\section{Table 1}

Size and zeta-potential of micelles and liposomes (Mean \pm S.D., $n=3$ )

\begin{tabular}{|c|c|c|}
\hline Formulation & Size $(\mathbf{n m})$ & Zeta potential $(\mathbf{m V})$ \\
\hline PEG $_{2000}$-PE micelles & $17.2 \pm 0.4$ & $-6.8 \pm 1.7$ \\
\hline NCL-240 micelles & $17.8 \pm 1.1$ & $-3.9 \pm 1.4$ \\
\hline 0.67 mol\% Tf-micelles & $38.2 \pm 5.2$ & $-31.4 \pm 0.9$ \\
\hline 1.34 mol\% Tf-micelles & $45.8 \pm 5.6$ & $-37.5 \pm 1.3$ \\
\hline Plain liposomes & $144.2 \pm 3.7$ & $-42.3 \pm 6.6$ \\
\hline NCL-240 liposomes & $152.6 \pm 3.3$ & $-39.9 \pm 3.5$ \\
\hline 0.5 mol\% Tf-liposomes & $178.8 \pm 1.5$ & $-36.1 \pm 3.3$ \\
\hline 1 mol\% Tf-liposomes & $195.9 \pm 3.0$ & $-30.4 \pm 1.4$ \\
\hline 2 mol\% Tf-liposomes & $217.9 \pm 4.5$ & $-32.2 \pm 2.1$ \\
\hline
\end{tabular}

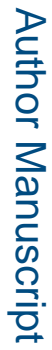

This table provides information regarding size and zeta potential characterization studies of lipid-based micelles and liposomes. 